\title{
Study of Herbal-Drug Interactions (HDIs) Using in Silico Methods - Mission (Im)Possible
}

\author{
Kaloyan D Georgiev* \\ Department of Pharmaceutical technologies, Medical University, Bulgaria
}

*Corresponding author: Kaloyan Georgiev, Department of Pharmaceutical technologies, Faculty of Pharmacy, Medical University "Prof. Dr. Paraskev

Stoyanov", Varna, Bulgaria.

Received Date: November 06, 2019

Published Date: November 21, 2019

\section{Opinion}

Studying herb-drug interactions (HDIs) is extremely important for the clinical practice, as many patients with chronic diseases taking a number of conventional medicines, also take phytomedicines, decoyed by advertising, without realizing that these herbal products in some cases can be harmful instead of beneficial. Herbal extracts contain a very large range of substances (more than 200 sometimes) that can affect both the pharmacokinetic and the pharmacodynamic characteristics of the conventional therapy. However, can these interactions be evaluated by in vitro and in silico methods in order to be highly informative without major clinical trial costs? What are the difficulties that must be taken into account in order to overcome the trade-offs that are being made? This mini-review aims to address briefly these issues and and the difficulty of building the models and the simulations with herbal drugs. More patients suffering from chronic diseases are taking herbal medicines, claiming them to be effective agents with little or negligible undesirable effects. Many of them, however, could lead to important clinical herb-drug interactions (HDIs) [1-3]. Herbal extracts contain many biologically active substances, with specific pharmacological characteristics, which in some cases may act in different directions. The most common form of interaction is the inhibition of the activity of the cytochrome enzymes and, therefore, this mechanism will be largely considered [4]. Evaluation of possible drug interactions is most commonly evaluated in vitro, using isolated liver microsomes or recombinant forms of cytochrome enzymes $[5,6]$. The mechanism of the inhibition can be - reversible competitive, and irreversible, mechanism-based. The values of inhibitory concentration $50\left(\mathrm{IC}_{50}\right)$ are calculated, which subsequently, after clarifying the mechanism of action, recalculate the inhibition constants $\left(\mathrm{K}_{\mathrm{i}}\right.$ - for reversible, $\mathrm{K}_{\mathrm{I}}$ and $\mathrm{k}_{\text {inact }}$ - for irreversible inhibition). So far, the processes are not complicated to perform. According to the guidelines for studying drug interactions, basic static models are used to evaluate the potential of the new drug to cause drug-drug interactions (DDIs). However, in addition to the inhibitory constant, it is necessary to know the plasma concentration of the drug, in the case of plant extracts and fractions, because of the many substances in them, which is a problem. Alternatively, each plant extract exhibiting inhibitory potential on CYP3A4 can be tentatively calculated for the potential for herb-drug interactions with substrates of enterocytic CYP3A4 isoenzymes, using the basic static equations, where plasma concentrations are exchanged with concentrations in GIT, namely:

$$
\frac{A U C_{i}}{A U C_{o}}=\frac{C L_{o}}{C L_{i}}=1+\frac{\left[I_{g}\right]}{K_{i}}
$$

where $A U C_{i}$ and $A U C_{o}$ are the values of the areas under the curves with inhibitor and no inhibitor respectively, $C L_{i}$ and $C L_{o}$ are the values of clearance with inhibitor and no inhibitor respectively, $\left[I_{g}\right]$ is the concentration of inhibitor, in this case the plant extract in the gastrointestinal tract, and $K_{i}$ is the enzyme inhibition constant by reversible competitive inhibition. The inhibitor concentration in the intestinal lumen, $\left[I_{g}\right]$, was calculated as follows: $\left[I_{g}\right]=$ Taken dose of the plan extract $/ 250 \mathrm{ml} .250 \mathrm{ml}$ is assumed to be the volume in which the entire dose is dissolved, and this dissolution is immediate. In addition, it is suggested that the full dose becomes available for intestinal enzymes. Consequently, it provides a high result for $\left[I_{g}\right]$. In other words, worst case scenarios are considered.

However, how can we evaluate the risk of HDIs which have affected hepatocyte cytochrome enzymes, without resorting to in vivo studies? Using in silico techniques, could we simulate pharmacokinetic behavior in the body and determine the potential for herbal extract-drug interactions? This is the right way, in the case of pure substances. Next steps involve using mechanistic static models or more comprehensive dynamic PBPK (Physiology-based 
pharmacokinetic) models. For the last one, pharmacokinetic data, such as bioavailability, volume of distribution and clearance, are needed. All new compounds, with inhibitory potential in vitro, are all candidates for DDI simulations in virtual populations to evaluate a risk. Software platforms that can perform this, and are widely used, are e.g. SimCYP, GastroPlus and PKsim. But how can we use them with plant extracts and isolated fractions? One of the ways is, when there is a major component in the extract or the isolated fraction, that is responsible for the activity, e.g. the inhibition of the cytochrome enzyme, and there is sufficient literature for its pharmacokinetic behavior. Of course, a number of assumptions need to be made, which, in principle, are not sufficiently correct, but would allow such models to be drawn up. For example, our studies with methylxanthine fractions isolated from Bancha and Pu-erh tea leaves showed little inhibitory effect on CYP3A4 isoenzymes [7]. To simulate possible drug interactions, we have used a simulator (in our case ADMEWORKS DDI, available at http:// www.fqs.pl/chemia/produkty/admeworks-ddi-simulator), and we have accepted data for the main component, which we have determined in the methylxanthine fractions, namely caffeine. The results of the simulations have showed little effect on the pharmacokinetic parameters of the classical substrate for the CYP3A4 enzyme evaluation - midazolam. Unfortunately, however, in most of the extracts there are many active components from different groups with different pharmacological properties. This is the case with green tea extracts as well. Their composition includes a large range of active substances, and in addition to the mentioned methylxanthins, catechins (e.g. epigallocatechin-3-gallate, EGCG) are included as well. Assessment by individual fractions, on the one hand, eliminates the "noises" of the other components, but on the other hand, it fails to assess the true effect or risk, as these components are taken at the same time and in the most cases mutually potentiate and support each other's activities. Even minimal changes in the composition could have an effect on both the pharmacokinetic and the pharmacodynamic behavior of the plant extract or the isolated fraction $[8,9]$.

Alternatively, another way is, for example, to analyze the major component(s) in the plant extracts or fractions and, if pharmacokinetic data are lacking, to generate them using the socalled ADME predictors, for example free platforms as swissADME or pkCSM. After receiving the data from the predictors, some basic calculations and assumptions have to be made as well. Anyway, this is eligible alternative way to reach the needed data to conduct the dynamic PBPK simulations. Assessing the potential for drug interactions of each new drug is a prerequisite for approval by the regulatory agencies. For nutritional supplements, it is only advisable presently. Unfortunately, these approaches can only guide us to possible interactions between herbal extracts and medicines. If in vitro and in silico methods do not show a risk of such interactions, then it probably does not exist. In the other case, if they show a risk, then that risk should be investigated in vivo, to be confirmed or rejected.

\section{Acknowledgement}

None.

\section{Conflict of Interest}

No conflict of interest to declare.

\section{References}

1. Hu Z, Yang X, Ho PC, Chan SY, Heng PW, et al. (2005) Herb-drug interactions: a literature review. Drugs 65(9): 1239-1282.

2. Chen XW, Sneed KB, Pan SY, Cao C, Kanwar JR, et al. (2012) Herb-drug interactions and mechanistic and clinical considerations. Curr Drug Metab 13(5): 640-651.

3. Borse SP, Singh DP, Nivsarkar M (2019) Understanding the relevance of herb-drug interaction studies with special focus on interplays: a prerequisite for integrative medicine. Porto Biomed J 4(2): e15.

4. Michalets EL (1998) Update: clinically significant cytochrome P-450 drug interactions. Pharmacotherapy 18(1): 84-112.

5. Venkataramanan R, Komoroski B, Strom S (2006) In vitro and in vivo assessment of herb drug interactions. Life Sci 78(18): 2105-2115.

6. Georgiev KD, Radeva Ilieva M (2019) In vitro inhibitions of CYP2C9 and CYP3A4 by fractions isolated from Goji berry (Lycium barbarum) fruits. Scripta Scientifica Pharmaceutica 6(1): 17-21.

7. Georgiev KD, Radeva Ilieva M, Stoeva S, Zhelev I (2019) Isolation, analysis and in vitro assessment of CYP3A4 inhibition by methylxanthines extracted from Pu-erh and Bancha tea leaves. Sci Rep 9(1): 13941.

8. Georgiev KD, Slavov IJ, Iliev IA (2019) In vitro evaluation of combination effects of doxorubicin with methylxanthine fractions isolated from Bancha and Pu-erh teas against breast cancer cells. Int J Basic Clin Pharmacol 8: 2167-2171.

9. Georgiev KD, Slavov IJ, Iliev IA (2019) Synergistic Growth Inhibitory Effects of Lycium barbarum (Goji berry) Extract with Doxorubicin against Human Breast Cancer Cells. J Pharm Pharmacol Res 3: 51-58. 